\title{
Tracer Pharmaceuticals Availability and Distribution Trends Prior to and During the Covid-19 Pandemic: A Comparative Study
}

\author{
Tafesse Gizaw (D)' \\ Awol Jemal ${ }^{2}$ \\ Tadesse Gudeta $\mathbb{D}^{2}$ \\ 'Quantification and Market Shaping \\ Office, Ethiopian Pharmaceuticals Supply \\ Agency, Jimma, Ethiopia; ${ }^{2}$ Department of \\ Social and Administrative Pharmacy, \\ School of Pharmacy, Institute of Health, \\ Jimma University, Jimma, Ethiopia
}

Background: Coronavirus disease 2019 (Covid-19) has had an unprecedented impact on the health products delivery system. To maintain routine health services, tracer pharmaceuticals (TPs) should always be available and accessible in healthcare institutions. Thus, this study aimed to explore the influence of the Covid-19 on TPs availability and distribution trends in the context of the Covid-19 pandemic in Ethiopia.

Methods: An institution-based comparative study was undertaken on 25 TPs in the pharmaceutical supply agency. From the agency's logistical data, we collated the amount of TPs ordered, received, and distributed, as well as the stock status, by using an observation checklist. The collated data were recorded in Microsoft Excel and transferred to SPSS for analysis. A frequency and percentage analysis were performed to evaluate the fill rate, product availability, and stock-out duration. A dependent $t$-test was used to compare if there was a significant mean difference in TPs distribution before and after the Covid-19 at a p-value less than $5 \%$.

Results: Before Covid-19, 18 (72\%) of the TPs were refilled above $80 \%$ of amount ordered. After the pandemic, however, the fill rate dropped to $48 \%$. The availability of TPs during the Covid-19 was $60 \%$. During the six months of Covid-19, the average stock-out duration for all TPs was 34.68 days, with Cimetidine $200 \mathrm{mg} / \mathrm{mL}$ injectable (line-item fill rate $=0$, days out of stock $=180$ days) being the most stocked-out item. Tracer pharmaceuticals distribution before Covid-19 ( $\mathrm{M}=81,317, \mathrm{SD}=139,274)$ were significantly different after the pandemic $(\mathrm{M}=60,212, \mathrm{SD}=94,966)$ at the $5 \%$ significance level $(\mathrm{t}(24)=2.289, \mathrm{p}=0.031)$. Conclusion: This survey reveals how Covid-19 has influenced service providers' logistics systems. Following the pandemic, the line fill rate fell below half with a three-fifths availability of products. To effectively manage supply chain disruptions experienced in Covid-19 and beyond, the agency in collaboration with stakeholders should make an effort to enhance end-toend inventory visibility, establish a resilient logistics system, strengthen domestic manufacturers' capabilities, and develop contingency plans with supplying partners.

Keywords: Covid-19, tracer pharmaceutical, availability, distribution, pharmaceutical supply agency, Ethiopia

\section{Introduction}

The coronavirus disease 2019 (Covid-19) outbreak was declared a global pandemic by the World Health Organization in March 2020. The pandemic has overwhelmed havoc all over the globe since its inception in Wuhan, China, in December 2019. ${ }^{1-3}$ Globally, over 221 million confirmed Covid-19 cases have been reported, affecting over 220 nations, regions, and territories. As of September 5th, 2021, the pandemic
Correspondence: Tafesse Gizaw Quantification and Market Shaping Office, Ethiopian Pharmaceuticals Supply Agency, PO Box 359, Jimma, Ethiopia

Email tafessegizaw@gmail.com 
has afflicted all African countries, resulting in 7,972,396 total cases and 199,438 deaths (case fatality rate of $2.50 \%) .{ }^{4}$ The cost of the pandemic has been disastrous on human health, social networks, and economic subsistence in many societies. ${ }^{5,6}$ African countries, in particular, lack the capacity, performance, and preparedness to respond to outbreaks while maintaining routine health services. $^{7}$

Having a knock-on effect on the prevention, diagnosis, care, and treatment of other diseases, better access to drugs and medical equipment may be compromised by the pandemic. ${ }^{8}$ Owing to this, other infectious diseases such as HIV/AIDS, tuberculosis (TB), and malaria were pushed to the sidelines, despite they are still very real problems, particularly among the most vulnerable communities. ${ }^{9,10}$ Failure to adequately provide treatments for these diseases and infections may result in avoidable deaths and resistant strains. In Ethiopia, HIV/AIDS, TB, malaria, noncommunicable diseases, injuries, and vaccine-preventable diseases like measles have been among the leading causes of morbidity and mortality over the last five years. ${ }^{11,12}$

A pharmaceutical supply chain is a multifaceted process that requires a continuous flow of products, orders, information, and funds between the points of production and sites of consumption to conform to customer requirements. ${ }^{13}$ The Covid-19 pandemic has resulted in more widespread public and global lockdowns, as well as the closure of many manufacturing units and businesses, exchange and transportation restrictions, and other logistical procedures. Taking together, the pandemic has had an impact on major countries that are the sole producers of raw materials, intermediate products, and consumer goods, causing supply chain disruption. ${ }^{8,10,14,15}$

Tracer pharmaceuticals (TPs) are essential medicines that address the population's priority health problems and their treatments. ${ }^{16}$ To provide consistent, high-quality care, and manage top community pressing problems, the TPs should be available and accessible at all times at healthcare institutions. ${ }^{17-20}$ In Ethiopia, TP availability and access are core indicators of supply chain performance. It is a proxy indicator of a health program's ability to meet clients' needs with a full range of products and services. $^{12,21}$

Furthermore, the country faces challenges such as a nationwide shortage of essential pharmaceuticals, inadequate cold chain infrastructure, limited coverage of diagnostic and treatment facilities, ineffective stock management, and a lengthy procurement process. ${ }^{11,21}$ This is a major concern in a country's health sector transformation plan that envisions improving healthcare product management systems to ensure uninterrupted availability and accessibility to address health problems.

The government of Ethiopia has taken public health and political measures as part of worldwide efforts to contain the virus from spreading. A state of emergency was declared, and border closures, as well as travel and trade restrictions between countries, resulted in a considerable reduction in the number of essential medicines, making it difficult for healthcare facilities to get the products they need. ${ }^{22-24}$

In Ethiopia, the pharmaceutical supply agency (EPSA) has been delivering essential pharmaceuticals to all public healthcare facilities (HCFs). Currently, there are 19 EPSA hubs within a 160 to 300 kilometer radius of one another, with the main branch in the country's capital. The HCFs use the report and requisition forms (RRF) to report their consumption and to request resupply quantities every two months. This is the primary method by which HCFs acquire health program pharmaceuticals (most notably antiretrovirals (ARVs), TB medicines, antimalarials, family planning contraceptives (FPC), vaccines, maternal and child health $(\mathrm{MCH})$ items) as well as budget pharmaceuticals (revolving drug fund - RDF). ${ }^{21,25}$

The Covid-19 pandemic may have long-lasting implications for how people work and supply chains operate. To leverage the effect and recover from the post-disruption condition, the pharmaceutical supply chain requires preparedness, response, and recovery capabilities. To this end, understanding the influence of the Covid-19 on the availability of TPs and their subsequent distribution is essential for efficiently managing supply chain disruptions in the context of such respiratory pandemics as well as other events that may happen in the future. Thus, this study aimed to explore the influence of the Covid-19 pandemic on TPs availability and distribution trends in the pharmaceutical supply agency in the context of the coronavirus outbreak in Ethiopia.

\section{Methods}

\section{Study Setting, Design, and Period}

The study took place at EPSA's Jimma hub. Jimma is about 350 kilometers from Addis Ababa, Ethiopia's capital. The hub serves more than ten million people in the country. With the prime responsibility for managing the healthcare supply chain in the western region, the hub 
plays a critical role in forecasting, inventory management, storage, and distribution of pharmaceuticals to HCFs. More than 349 public HCFs (27 public hospitals and 322 health centers), three private hospitals, four community pharmacies, eight red-cross drug stores, as well as different private pharmacy outlets and drug vendors were served by the hub. In addition to paper-based records, the agency uses electronic health commodity management information system (HCMIS) software to manage logistics information and warehouse operations. We used a retrospective observational analysis with a comparative approach between September 1, 2020, and November 6, 2020.

\section{Population}

The source population included all healthcare commodities managed by the EPSA Jimma hub and the HCMIS. The study population included all tracer commodities ordered, received, and distributed by the hub, the HCMIS, and the logistics records (bin-cards, RRFs, goods receiving and issuing vouchers) used to track commodity transactions before the first occurrence of Covid-19 (between September 2019 and February 2020) and after the pandemic's occurrence (between March and August 2020). In Ethiopia, annual stock verification is usually done in July. During this month, all stock transactions are closed (frozen). To account for this, the analysis used transaction data through September 2020 instead of July 2020. The first Covid-19 case was reported in Ethiopia on March 13, 2020. The study compared six (6) months of transaction data before and after the outbreak. Thus, the analysis considers tracer commodities that are ordered, received and issued (distributed) in the hub as well as TPs stock status on the day of data collection.

\section{Sampling Procedure}

Taking into account medicine availability indicators and tracer lists in use in Ethiopia, ${ }^{12}$ the study examined all 13 health program specific tracer commodities $(\mathrm{ARVs}=2$, $\mathrm{TB}$ drugs $=2, \mathrm{MCHs}=3$, antimalarials $=3, \mathrm{FPCs}=2$, vaccine $=1$ ), and 12 RDF pharmaceuticals managed by the hub. Overall, the 25 tracer pharmaceuticals are priority treatment choices for the most common morbidities and/ or required for preventive or diagnostic health services.

Every other month, the EPSA hubs use RRF to report and order commodities from the main branch to maintain proper stock levels and prepare commodities for redistribution. $^{25}$ Thus, a hub should send a total of six RRFs per year and can receive six different types of orders in the same year. As a result, we reviewed six RRFs, as well as all goods receiving and issuing vouchers, to determine the amount ordered, received, and distributed over the year. The physical inspection, accompanied by the HCMIS transaction data, was used to evaluate the stock status of TPs (product availability or days out of stock).

\section{Data Collection Instrument and Procedure}

We used an observation checklist modified following standard recommendations, such as the logistics indicators assessment tool, ${ }^{26}$ the Ethiopian Ministry of Health strategic framework, ${ }^{12}$ and world health organization harmonized monitoring and evaluation indicators, ${ }^{27}$ to collect the relevant data. The observation checklist included closed-ended questions about the amount of TPs ordered, received, and distributed, as well as the stock status on the day of data collection. On the other essential medicines, the observation checklist was tested for its content validity. During data collection, a directive issued by the Public Health Institute (Ethiopian) for the prevention and control of the Covid-19 pandemic was followed. A trained data collector (a pharmacy professional) was enlisted to help with data gathering under the supervision of the lead investigators (Additional file).

\section{Data Processing and Analysis}

The collated data were coded and abstracted into Microsoft Excel 2016 and thereafter transferred into the Statistical Package for the Social Sciences (IBM Corp., Armonk, NY, USA) version 26 for analysis. A descriptive frequency and percentage analysis were performed to evaluate the line fill rate, product availability, and distribution trends before and after the Covid-19 pandemic. The following formulas were used to compute current product availability, stock out duration, and line fill rate of TPs: ${ }^{12,26,27}$

$$
\begin{aligned}
& \text { No of tracer pharmaceuticals available } \\
& \text { Product availability }=\frac{\text { at the time of data collection }}{\begin{array}{l}
\text { The number of tracer } \\
\text { pharmaceuticals considered }
\end{array}} \\
& * 100 \% \\
& \begin{aligned}
& \text { Average stock }- \text { out duration }= \frac{\text { product was not available }}{\text { The number of tracer }} \\
& \text { pharmaceuticals reviewed } \\
& * 100 \%
\end{aligned}
\end{aligned}
$$




$$
\text { Line }- \text { item fill rate (LIFR) }=\frac{\begin{array}{l}
\text { The amount (in quantity ) } \\
\text { of tracer item received }
\end{array}}{\begin{array}{l}
\text { The amount }(\text { in quantity }) \\
\text { of tracer item ordered }
\end{array}} * 100 \%
$$

Line fill rate for all TPs

$$
=\frac{\text { The number of TPs filled correctly }(\text { LIFR } \geq 80 \%)}{\text { The number of tracerpharmaceuticals reviewed }} * 100 \%
$$

A dependent (paired-sample) $t$-test was used to compare if there is a significant mean difference between TPs distribution before and after the Covid-19. A p-value of less than $5 \%$ was considered statistically significant. ${ }^{28}$ The results were presented using texts, tables, and graphs.

\section{Operational Definitions}

Health program pharmaceuticals: Products distributed freeof-charge to healthcare facilities by the EPSA hubs, such as ARVs, TB-drugs, antimalarials, family planning contraceptives, maternal and child health products, and vaccines.

Revolving drug fund pharmaceuticals: Products purchased by HCFs through the "healthcare financing system" or "Ministry allocated budgets" from EPSA hubs.

\section{Results}

\section{The Line-Item Fill Rate Analysis}

In this study, the line fill rate is operationalized as the proportion of TPs ordered by the hub from the main branch in a year that is filled at least $80 \%$ in terms of the quantities requested. Prior to the start of Covid-19, 18 ( $72 \%$ ) of the line items were refilled at least $80 \%$ by the main branch. After the pandemic, however, the fill rate dropped to $48 \%$. When the TPs were disaggregated by category, the line fill rate for health program products fell from full supply to $61.5 \%$ (Figure 1). Revolving drug fund (RDF), on the other hand, was relatively low (33.3\%) following the pandemic (Figure 2).

\section{Current Product Availability}

The study assessed the stock availability of 25 baskets of tracer pharmaceuticals on the day of data collection and the duration of stock-outs over the six months preceding the survey. The availability of TPs during the Covid-19 pandemic was $60 \%$. Ten of the twenty-five (40\%) TPs evaluated had stock-outs on the day of data collection.

\section{The Average Duration of Stock-Outs at EPSA Warehouse}

This indicator assesses the average duration of stock-out of TPs expected to be issued (distributed) by the hub. In the six months preceding data collection, Cimetidine 200mg/ $\mathrm{mL}$ injectable, an RDF item, had a stockout duration of 180 days. Health program commodities stocked out for an average of 9.07 days in the agency, with Etonogestrel $68 \mathrm{mg}$ subdermal implant (Implanon $^{(\mathbb{B}}$ ) with insertion/ removal accessory (39 days) being the most stocked-out item. For all TPs evaluated, the average stock-out duration was 34.68 days.

\section{Distribution Trends of Tracer Pharmaceuticals}

EPSA has been delivering health program commodities directly to HCFs on a bimonthly basis. The facilities, on the other hand, are expected to purchase RDF products from affiliated hubs. In this study, the distribution trend of health program pharmaceuticals has variations in demand with a slight increase in distribution (Figure 3), whereas the distribution of RDF commodities has decreased dramatically (Figure 4).

\section{Comparison of Tracer Pharmaceuticals Distribution Trends}

The data was collated over six (6) months before and following the Covid-19 pandemic. The dependent (sample-paired) $t$-test would test the null hypothesis that the average mean of the TPs distribution was the same before and after the pandemic. However, TPs distribution prior to Covid-19 $(\mathrm{M}=81,317, \mathrm{SD}=139,274)$ were significantly different after the pandemic $(\mathrm{M}=60,212, \mathrm{SD}=94,966)$ at the $5 \%$ significance level $(\mathrm{t}(24)=2.289, \mathrm{p}=0.031$ ) (Tables 1 and 2).

\section{Discussion}

In this study, we evaluated how the Covid-19 pandemic affected tracer product availability and distribution trends in the Ethiopian pharmaceutical supply agency in the context of the country's coronavirus outbreak. We compared the fill rate, distribution trends, and product availability before and during the Covid-19. Line fill rate measures the ability of inventory to meet customer needs. Having a profound impact on operational performance, line fill rate can be used as a practical measure to benchmark the order fulfillment performance of suppliers over a specific 
Oxytocin Injection $10 \mathrm{IU} / \mathrm{ml}$ in $1 \mathrm{ml}$ Injection, of 50 Pack

Ferrous sulphate + Folic Acid (200mg + $0.4 \mathrm{mg}$ ) Tablet, $10 \times 10$ Pack

Oral rehydration salts Powder, in sachet

DTP-Hib-Hep (Pentavalent) Vaccine, in 1 dose

Artesunate $60 \mathrm{mg}$ vial injection, in $1 \mathrm{ml}$ Ampoule

Artemether + lumefanthrine $(20+120) \mathrm{mg}, 24 \times 30$ Tablet

Rapid Diagnostic Test (MRDT), PF\&PV Cassette, in 25 tests

Etonogestrel 68mg Capsule (Subdermal Implant) with insertion/removal accessory kit

Medroxyprogesterone Acetate $-150 \mathrm{mg} / \mathrm{ml}$ in $1 \mathrm{ml} \mathrm{Vial} \mathrm{-}$ injection with $21 \mathrm{G}$ needle

RHZ (75mg+50mg+150mg) - Tablet (of $28 \times 3$ Pack)

RHZE (150mg+75mg+400mg+275mg) + RH (150mg+75mg)- Tablet, (in Kit)

HIV Rapid test kit assay 1 - Statpack - of 20 tests

Lamivudine (3TC) + Dolutegravir (DTG) + Tenofovir (TDF) $-(300 m g+50 m g+300 m g)-30$ Tablets

$\square$ Line fill rate during Covid-19 [Mar to Aug, 2020]
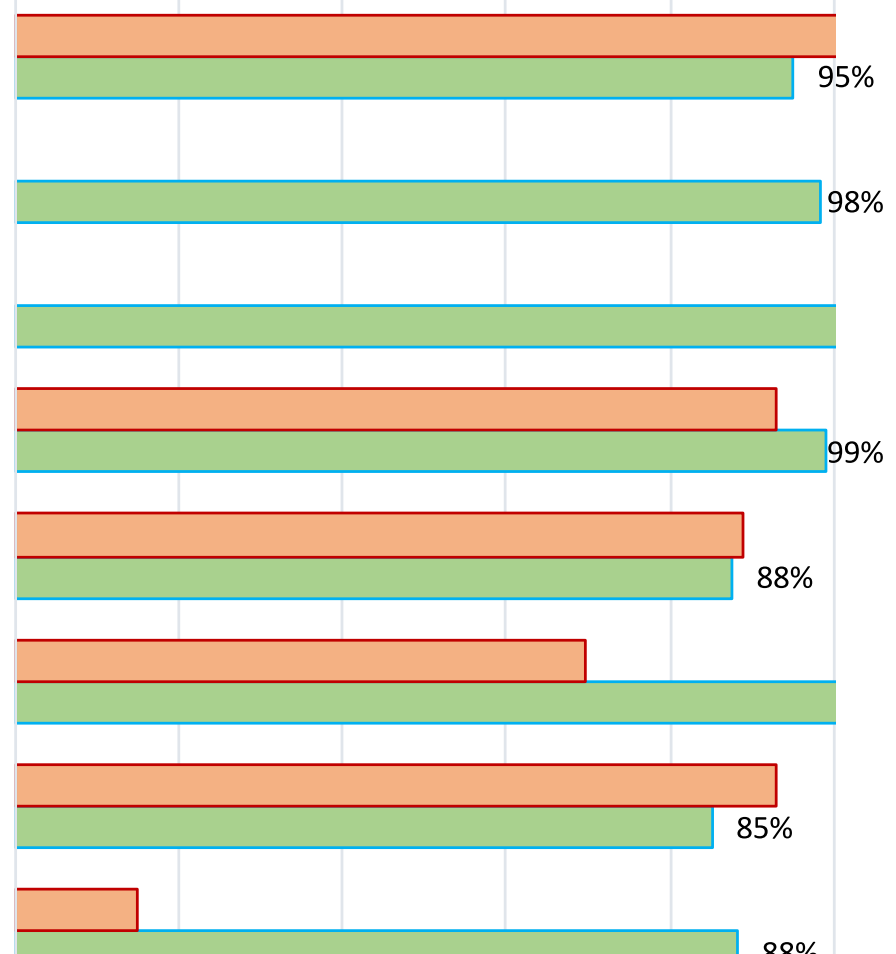

$88 \%$
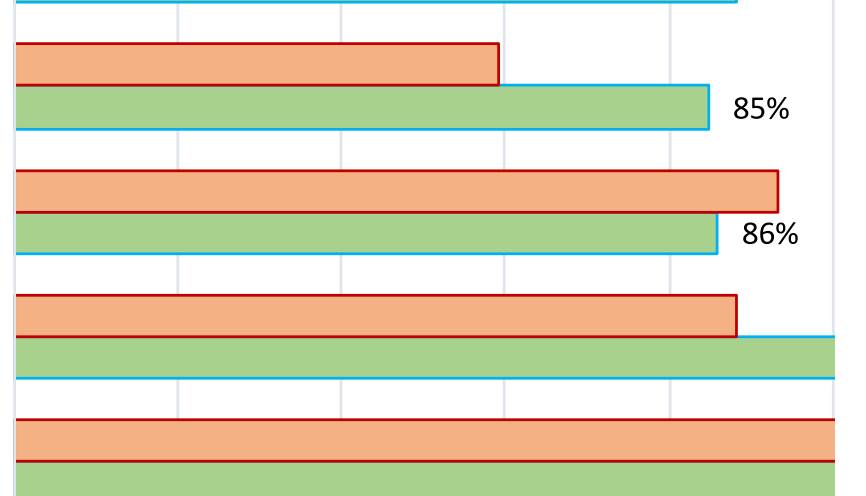

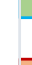

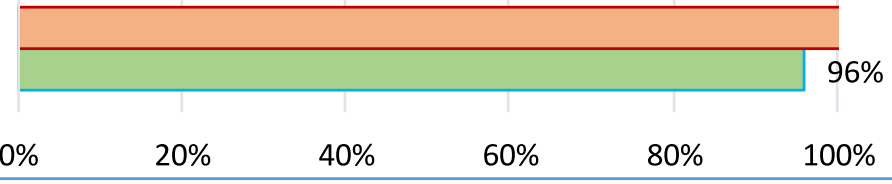

$\square$ Line fill rate before Covid-19 [Sep 2019 to Feb, 2020]

Figure I Line-item fill rate for tracer health program pharmaceuticals before and during COVID-I9.

period. Typically, a flawless fill rate is $100 \% .{ }^{12,26,27}$ In the Ethiopian context, however, an order is filled correctly when the supplied quantities correspond to at least $80 \%$ of the ordered quantities. The following are possible reasons for the fill rate to be set at $80 \%$. Exaggerated requests or arithmetic (typo) errors will be adjusted reasonably by the supplier, the requested quantity may come in one pack size, pilferage can occur during shipment, and the warehouse may intentionally ration limited quantities on purpose.

EPSA has been implementing the integrated pharmaceutical logistics system to develop unified healthcare at all levels of the supply chain, and to offer timely data for logistics decision-making. In this system, the main branch is in-charge of resupplying hubs, and the hubs are incharge of HCFs. ${ }^{25}$ In the current study, following the 


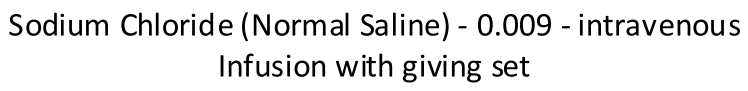

Phenobarbitone (Phenobarbital) - 100mg - Tablet

Paracetamol - 500mg - Tablet

Glove examination latex - all sizes (Large, Medium or small)

Ciprofloxacin - 500mg - Tablet

Cimetidine $-200 \mathrm{mg} / \mathrm{ml}$ injectable

Ceftriaxone - $1 \mathrm{~g}$ in vial - powder for injection with $10 \mathrm{ml}$ diluent

Amoxi cillin - 500mg - Capsule

Adrenaline (Epinephrine) - $0.1 \%$ in $1 \mathrm{ml}$ ampoule, injectable

Metformin - 500mg - Tablet

Enalapril Ma leate - $5 \mathrm{mg}-$ Ta blet

Hydrochlorothiazide - $25 \mathrm{mg}$ - Ta blet

$0 \%$

- Line fill rate during Covid-19 [Mar to Aug, 2020]
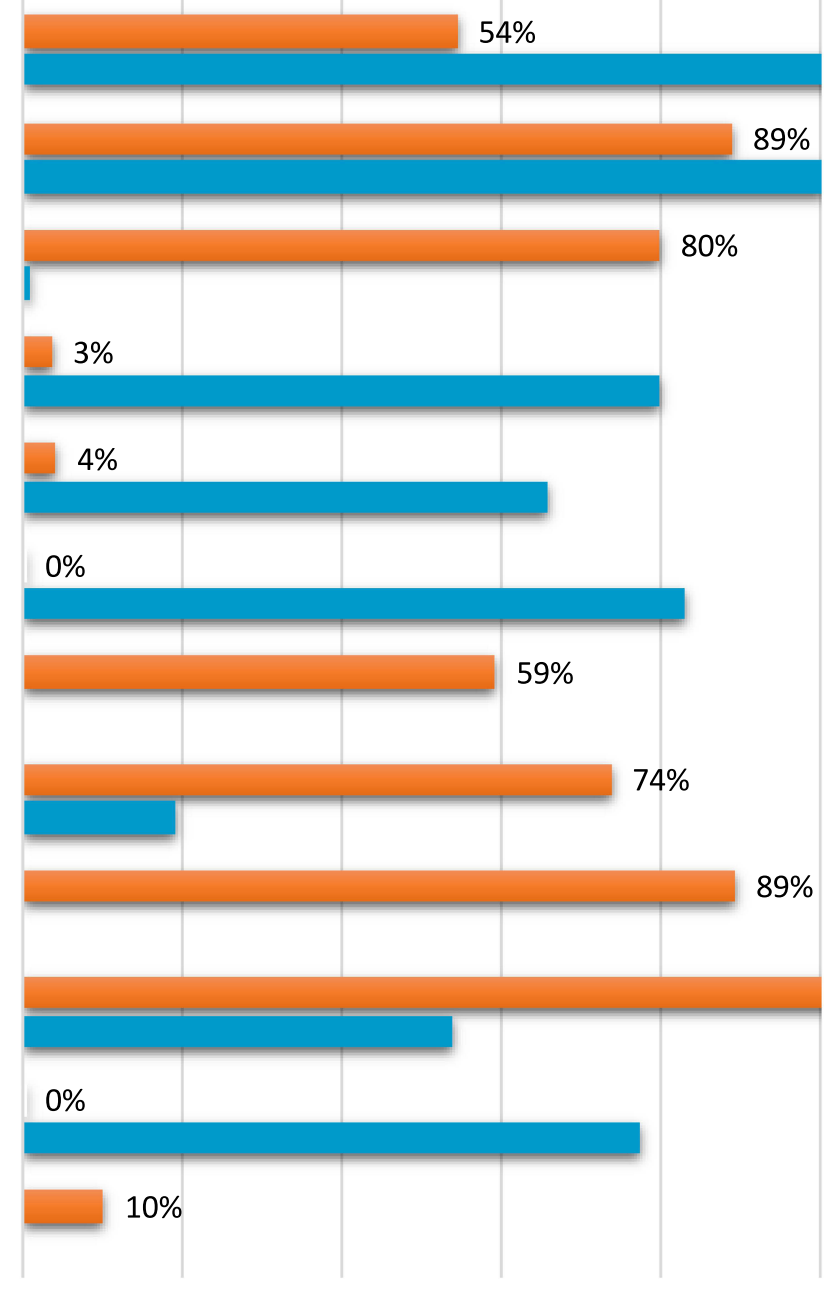

$20 \%$

$40 \%$

$60 \%$

$80 \%$

$100 \%$

Figure 2 Line-item fill rate for tracer RDF pharmaceuticals before and during COVID-19.

Covid-19 pandemic, the line fill rate dropped from $72 \%$ to $48 \%$ for the 25 TPs placed by the hub. This demonstrates that Covid-19 has had an impact on the fill rate of tracer items from the supplier and subsequent distribution. Owing to the pandemic's disruption and possible difficulties in acquiring products, border closures, as well as, the government proposed isolation measures, the pandemic has severely affected the supply chain. This study is comparable to studies conducted in Namibia, ${ }^{29}$ Saudi Arabia, ${ }^{30}$ and Nigeria. $^{31}$

Before the pandemic, most companies around the world were implementing lean supply chains, with an emphasis on cost optimization and just-in-time delivery. Thereby, the companies are left without a buffer and flexibility to absorb delays and interruptions in the supply chain. ${ }^{13,32}$ However, a resilient supply chain can withstand or even prevent the impact of supply network disruptions and can swiftly recover from them by implementing supply chain flexibility. ${ }^{33}$ The EPSA uses a Maximum-Minimum (Maxmin) inventory control policy. ${ }^{21,25}$ The maximum inventory a hub can hold in this policy is four months' stock, with a minimum level of two months and a reorder interval of two months. The agency thus does not have stock buffers to the extent that the Covid-19 pandemic is disruptive. The current study found that $60 \%$ of TPs were available after the Covid19 pandemic. For all TPs evaluated, the average stock-out duration was 34.68 days in the six months review period. This could imply that health logisticians in various facilities have struggled to maintain optimal stock levels and avoid stock-outs during the pandemic. As a result, the pandemic 


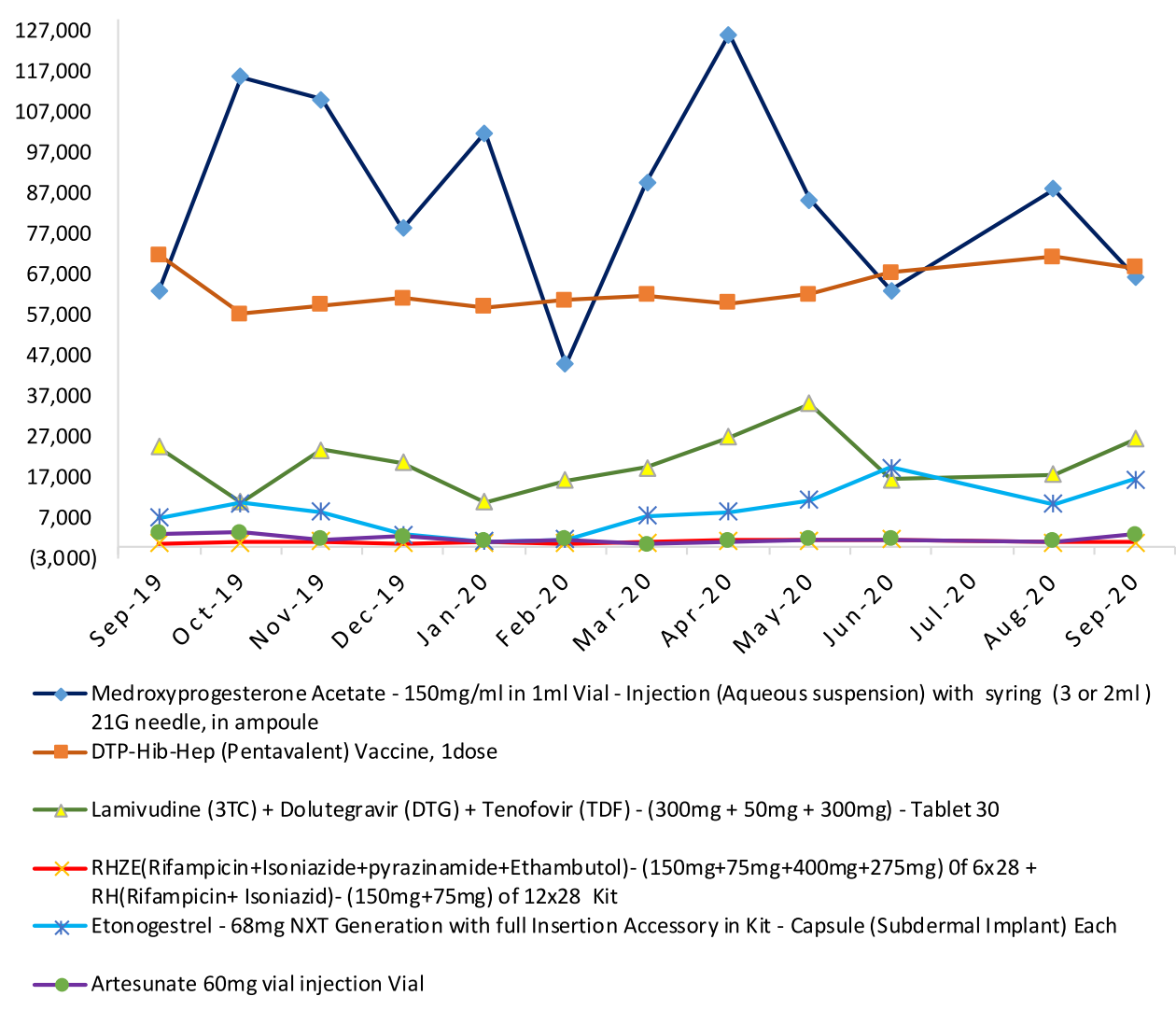

Figure 3 Distribution trends for tracer health program commodities.

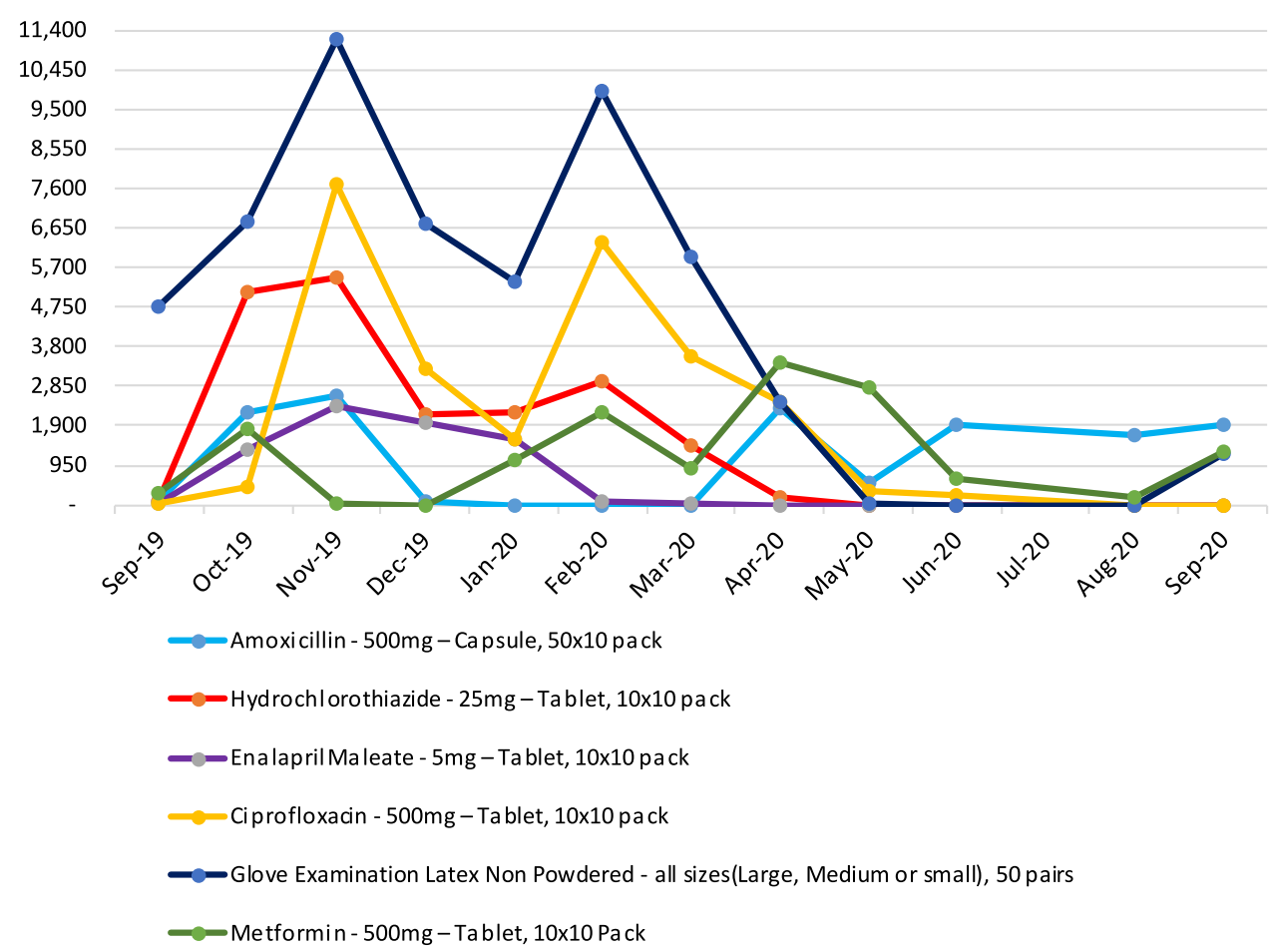

Figure 4 Distribution trends for tracer RDF commodities. 
Table I Paired Sampled Statistics Before and During the COVID-I 9 Pandemic

\begin{tabular}{|l|l|c|c|c|c|}
\hline \multicolumn{2}{|c|}{} & $\begin{array}{c}\text { Mean } \\
\text { (M) }\end{array}$ & $\mathbf{N}$ & $\begin{array}{c}\text { Std. } \\
\text { Deviation } \\
\text { (SD) }\end{array}$ & $\begin{array}{c}\text { Std. } \\
\text { Error } \\
\text { Mean }\end{array}$ \\
\hline $\begin{array}{l}\text { Pair } \\
\text { I }\end{array}$ & $\begin{array}{l}\text { Prior to Covid- } \\
19 \text { incidence } \\
\text { After the Covid- } \\
19 \text { pandemic }\end{array}$ & 81,317 & 25 & 139,274 & 27,855 \\
\hline
\end{tabular}

has exposed the flaws in the Max-min inventory control system in the EPSA hub. This entails the agency focusing on implementing agile working practices, establishing a resilient logistics system, strengthening domestic supply chain capabilities, regularly monitoring supplier performance, and developing contingency plans with supplying partners. ${ }^{15,29,34,35}$

Covid-19 has put an unprecedented demand on the healthcare products delivery system. Most nations rely on imports of drugs, raw materials, and equipment from countries outside their territory, most notably India and China. These countries also supply around $80 \%$ of the pharmaceuticals consumed in Ethiopia. ${ }^{36,37}$ Given the pandemic's expected supply and transportation difficulties, port congestion, lengthy customs clearances, or closure of a few treatment facilities (changed to Covid-19 treatment centers), demand for distribution is rapidly dropping. ${ }^{15}$ In this study, the distribution trend of health program pharmaceuticals has variations in demand with a slight increase whereas the RDF commodities have decreased dramatically. At the 5\% level of significance, the distribution of TPs prior to Covid-19 was significantly different after the pandemic $(\mathrm{t}(24)=2.289)$. This could be the fact that the majority of EPSA's RDF pharmaceuticals suppliers are from the China, the government's focus on the supply of drugs and equipment required to directly manage Covid-19, the health facility's reduced capacity to purchase essential medicines, or other logistical issues where China was unable to work at full capacity around the inception of Covid-19. ${ }^{8}$ In contrast, most pharmaceuticals for health programs (ARV, anti-TB \& antimalarial) are supplied from India and across Europe by international donors or charity organizations. ${ }^{10}$

Information visibility is integral to a successful supply chain network. Lack of information visibility along the supply chain impedes the ability to easily access and track realtime data. Product management in the event of a pandemic demands real-time supply chain information. ${ }^{13,32}$ In Ethiopia, almost all pharmaceutical distribution centers lack an end-toend view of the supply chain and the agility to predict today's unpredicted demand variations. ${ }^{11,38}$ Thus, inventory visibility across the extended supply network is limited at the hubs, contributing supply chain risks more challenging. To buffer typical supply variability and track delivery schedules, EPSA should leverage integrated information systems with suppliers, customers, and other supply chain nodes. This will provide diverse logisticians with a uniform perspective of the end-to-end supply chain visibility. ${ }^{14,39}$

\section{Limitations and Future Research}

During the study, considerable care was made to acquire the best information possible. However, a few limitations should be mentioned. Because the study focused solely on tracer medicines, the findings may not be generalizable to all medications. A more comparative study, in particular, is required to fully comprehend the findings. The availability of tracer pharmaceuticals applies solely to the day of data collection; no inferences of availability over time can be made based on this study. Indeed, comprehensive research is needed to appreciate the concept of continuous product availability. Logistics records were used to calculate the number of days of stock-outs. As a result, maintaining upto-date records is critical to their accuracy. Other longitudinal studies should use stock-out duration in conjunction with other indicators such as stocked-according-to-plan because facilities can ration supplies to avoid stock-outs.

Table 2 Mean Differences (t-test) of Tracer Pharmaceuticals

\begin{tabular}{|c|c|c|c|c|c|c|c|c|c|}
\hline & & \multicolumn{5}{|c|}{ Paired Differences } & \multirow[t]{3}{*}{$\mathbf{t}$} & \multirow[t]{3}{*}{$d f$} & \multirow{3}{*}{$\begin{array}{c}\text { Sig. } \\
\text { (2-Tailed) }\end{array}$} \\
\hline & & \multirow[t]{2}{*}{ Mean } & \multirow[t]{2}{*}{$\begin{array}{c}\text { Std. } \\
\text { Deviation }\end{array}$} & \multirow[t]{2}{*}{$\begin{array}{l}\text { Std. Error } \\
\text { Mean }\end{array}$} & \multicolumn{2}{|c|}{$\begin{array}{l}95 \% \mathrm{Cl} \text { of the } \\
\text { Difference }\end{array}$} & & & \\
\hline & & & & & Lower & Upper & & & \\
\hline $\begin{array}{l}\text { Pair } \\
1\end{array}$ & $\begin{array}{l}\text { Prior to Covid-19 incidence - After the } \\
\text { Covid-19 pandemic }\end{array}$ & 21,105 & 46,095 & 9219 & 2078 & 40,132 & 2.289 & 24 & 0.031 \\
\hline
\end{tabular}




\section{Conclusion}

This survey revealed how Covid-19 has influenced service providers' logistics systems. Following the inception of Covid-19, the line fill rate fell below half with a threefifths availability of products. For the TPs reviewed, the average stock-out time was more than a month. The distribution trend of selected products prior to Covid-19 was significantly different after the pandemic. These findings imply that a considerable number of the agency's healthcare facilities are more likely to experience tracer pharmaceuticals shortages amid Covid-19, which demands an improved pharmaceutical sector's preparedness and response for pandemic management. Thus, the agency in collaboration with stakeholders should prioritize enhancing end-to-end inbound inventory visibility, establishing a resilient logistics system, strengthening domestic manufacturers' capabilities, and developing contingency plans with supplying partners to manage supply chain disruptions experienced in Covid-19 and beyond.

\section{Implications of the Study}

To the best of our knowledge, this is one of the first studies to assess the line fill rate, current product availability, stock-out duration, and distribution trends in Ethiopia before and during the pandemic. Indeed, the study would help in understanding the public pharmaceutical sector's preparedness in the face of a Covid-19 pandemic. The findings could also assist pharmaceutical supply institutions, health program managers, or other stakeholders to better plan, manage, implement and evaluate logistics operations, as well as focus on developing a resilient logistics system to withstand potential supply chain disruptions in the future while maintaining routine services.

\section{Abbreviations}

ARV, antiretroviral therapy; Covid-19, coronavirus disease 2019; EPSA, Ethiopian pharmaceutical supply agency; FPC, family planning contraceptive; $\mathrm{HCFs}$, healthcare facilities; $\mathrm{MCH}$, maternal and child health; RDF, revolving drug fund; $\mathrm{RRF}$, report and requisition form; TP, tracer pharmaceutical.

\section{Data Sharing Statement}

This study includes all pertinent data. Additional information could be obtained from the corresponding author upon reasonable request.

\section{Ethical Consideration}

The study was conducted according to the guidelines of the Declaration of Helsinki. The ethical review board of Jimma University has approved the study. The authors also received permission and consent from the Ethiopian pharmaceutical supply agency. For this investigation, the authors used anonymised data. The data obtained from the observation were stored securely in a locked file cabinet throughout the study.

\section{Acknowledgments}

We would like to thank the EPSA-Jimma hub, as well as the data collectors, for providing us with necessary data/ information, for their kind cooperation, and assistance throughout the data collection process.

\section{Disclosure}

The authors report no conflicts of interest in this work.

\section{References}

1. Liang T. Handbook of COVID-19 prevention and treatment. Jack Ma Foundation and Alibaba Foundation; 2020. Available from: https:// asprtacie.hhs.gov/technical-resources/resource/7844/handbook-ofcovid-19-prevention-and-treatment. Accessed September 1, 2021.

2. African Union Member States. COVID-19 scientific and public health policy update. African Union/Africa CDC/WHO/AFRO; 2021. Available from: https://africacdc.org/download/policy-paperresearch-and-development-priorities-for-covid-19-in-africa/. Accessed September 1, 2021.

3. WHO. Report JW-CST-Convened Global Study of Origins of SARSCoV-2: China Part. 2021.

4. Centers for Disease Control and Prevention. Coronavirus disease 2019 (COVID-19); 2021. Available from: https://www.cdc.gov/coro navirus/2019-ncov/prevent-getti\%0Ang-sick/prevention.html. Accessed September 5, 2021.

5. Nkengasong JN, Ndembi N, Tshangela A, Raji T. COVID-19 vaccines: how to ensure Africa has access. Nature. 2020;586:197-199. doi:10.1038/d41586-020-02774-8

6. Teixeira JA. Corona exhaustion (CORONEX): COVID-19-induced exhaustion grinding down humanity. Curr Res Behav Sci. 2021;2:1-3. doi:10.1016/j.crbeha.2021.100014

7. Hagan JE, Ahinkorah BO, Seidu -A-A, et al. Africa's preparedness towards COVID-19 vaccines: demand and acceptability challenges. Curr Res Behav Sci. 2021;2(1-4):100048. doi:10.1016/j. crbeha.2021.100048

8. Roberton T, Carter ED, Chou VC, Stegmuller AR, Jackson BD, Tam Y. Early estimates of the indirect effects of the Covid-19 pandemic on maternal and child mortality in low-income and middle-income countries: a modelling study. Lancet. 2020;8(7):E901-E908.

9. Mohammed H, Oljira L, Roba KT, Yimer G, Fekadu A, Manyazewal T. Containment of COVID-19 in Ethiopia and implications for tuberculosis care and research. Infect Dis Poverty. 2020;9:1-8. doi:10.1186/s40249-020-00753-9

10. The Global Fund to Fight AIDS, Tuberculosis, and Malaria. COVID19 impact on supply chain logistics: assessment and recommendations. Sourcing \& management of health products; 2021. Available from: https://www.theglobalfund.org/en/covid-19/health-productsupply/. Accessed June 22, 2021. 
11. Federal Ministry of Health $(\mathrm{FMoH})$. Ethiopian Health Sector Transformation Plan (2015/16-2019/20). Federal Ministry of Health (FMoH); 2015.

12. The Ethiopian Federal Ministry of Health. National Pharmacy Service, Pharmaceuticals Supply Chain and Medical Equipment Management Monitoring and Evaluation Framework. The Ethiopian Federal Ministry of Health; 2019.

13. Chopra S, Meindl P. Supply Chain Management: Strategy, Planning, and Operation. Sixth ed. Edinburgh Gate: Pearson Education Limited; 2016.

14. Barman A, Das R, De PK. Impact of COVID-19 in food supply chain: disruptions and recovery strategy. Curr Res Behav Sci. 2021;2:1-5. doi:10.1016/j.crbeha.2021.100017

15. Kumar A, Luthra S, Kumar S, Kazançoğlu Y. COVID-19 impact on sustainable production and operations management. Sustain Oper Comput. 2020;1:1-7. doi:10.1016/j.susoc.2020.06.001

16. Schürmann M. Developing a List of Tracer Medicines for the Nepal Health Sector Programme-2: Literature Review and Recommendations. Kathmandu: Nepal Health Sector Support Programme, Ministry of Health and Population (GoN); 2012.

17. World Health Organization (WHO). Operational Package for Assessing, Monitoring and Evaluating Country Pharmaceutical Situations Guide for Coordinators and Data Collectors. World Health Organization (WHO); 2007

18. Management Science for Health. MDS-3: Managing Access to Medicines and Health Technologies. Arlington, USA: Management Science for Health; 2012.

19. Towards access 2030. World Health Organization (WHO) essential medicines and health products strategic framework 2016-2030. Geneva; 2017. Available from: http://www.who.int/medicines/publications/\% 0ATowards_Access_2030_Final.pdf. Accessed December 21, 2021.

20. Cantarero-Arévalo L, Holstein BE, Andersen A, Kristiansen M, Hansen EH. The availability of six tracer medicines in private medicine outlets in Uganda. J Pharm Policy Pract. 2014;7(18):1-8. doi:10.1186/s40545-014-0018-y

21. Ethiopian Pharmaceuticals Supply Agency. National Survey of the Integrated Pharmaceutical Logistics System (IPLS). Addis Ababa, Ethiopa: AIDSFree, and Pharmaceutical Supply Agency; 2019.

22. Nigussie H. The coronavirus intervention in Ethiopia and the challenges for implementation. Heal Commun Sect J Front Commun. 2021;6:1-12. doi:10.3389/fcomm.2021.562512

23. Zikargae M. COVID-19 in Ethiopia: assessment of how the Ethiopian government has executed administrative actions and managed risk communications and community engagement. Risk Manag Healthc Policy. 2020;13:2803-2810. doi:10.2147/RMHP.S278234

24. Gudina EK, Gobena D, Debela T, et al. COVID-19 in Oromia Region of Ethiopia: a review of the first 6 months' surveillance data. $B M J$ Open. 2021;11:1-9. doi:10.1136/bmjopen-2020-046764

25. Ethiopian pharmaceuticals Supply Agency (EPSA). Integrated Pharmaceuticals Logistics System (IPLS) in Health Facilities of Ethiopia. Ethiopian pharmaceuticals Supply Agency (EPSA); 2017.

26. USAID | DELIVER PROJECT, Task Order 1. Logistics Indicators Assessment Tool (LIAT). Arlington, Va.: USAID | DELIVER PROJECT, Task Order 1; 2008.

Journal of Multidisciplinary Healthcare

\section{Publish your work in this journal}

The Journal of Multidisciplinary Healthcare is an international, peerreviewed open-access journal that aims to represent and publish research in healthcare areas delivered by practitioners of different disciplines. This includes studies and reviews conducted by multidisciplinary teams as well as research which evaluates the results or conduct of such teams or healthcare processes in general. The journal

Submit your manuscript here: https://www.dovepress.com/journal-of-inflammation-research-journal
27. World Health Organization. Harmonized Monitoring and Evaluation Indicators for Procurement and Supply Management Systems: EarlyWarning Indicators to Prevent Stock-Outs and Overstocking of Antiretroviral, Antituberculosis and Antimalaria Medicines. Geneva, Switzerland: World Health Organization; 2011.

28. Yockey RD. SPSS Demystified: A Simple Guide and Reference. Third ed. New York and London: Routledge; 2018.

29. Tirivangani T, Alpo B, Kibuule D, Gaeseb J, Adenuga BA. Impact of COVID-19 pandemic on pharmaceutical systems and supply chain a phenomenological study. Explor Res Clin Soc Pharm. 2021;15:100037. doi:10.1016/j.rcsop.2021.100037

30. Aljadeed R, Alruthia Y, Balkhi B, et al. The impact of COVID-19 on essential medicines and personal protective equipment availability and prices in Saudi Arabia. MDPI Healthc. 2021;9(290):1-14. doi: $10.3390 /$ healthcare 9030290

31. Emmanuel Awucha N, Chinelo Janefrances O, Chima Meshach A, Chiamaka Henrietta J, Ibilolia Daniel A, Esther Chidiebere N. Impact of the COVID-19 pandemic on consumers' access to essential medicines in Nigeria. Am J Trop Med Hyg. 2020;103(4):1630-1634. doi:10.4269/ajtmh.20-0838

32. Nguyen TT. Wal-Mart's successfully integrated supply chain and the necessity of establishing the Triple-A supply chain in the 21 st ce. J Econ Manag. 2017;29(3):103-116. doi:10.22367/jem.2017.29.06

33. Mensah P, Merkuryev Y. Developing a resilient supply chain. Procedia Soc Behav Sci. 2014;110:309-319. doi:10.1016/j. sbspro.2013.12.875

34. Taggar R. Recovering supply chain disruptions in post-COVID-19 pandemic through transport intelligence and logistics systems: India's experiences and policy options. Front Future Transport. 2021;(2). doi:10.3389/ffutr.2021.660116

35. Goodarzian F, Ghasemi P, Gunasekaren A, Allah A, Ajith T. A sustainable-resilience healthcare network for handling COVID-19 pandemic. Ann Oper Res. 2021. doi:10.1007/s10479-021-04238-2

36. Federal Democratic Republic of Ethiopia Ministry of Health and Ministry of Industry. National Strategy and Plan of Action for Pharmaceutical Manufacturing Development in Ethiopia (20152025): Developing the Pharmaceutical Industry and Improving Access; 2015.

37. Martial NP, Sieleunou I. An appeal for large scale production of antiretroviral drugs in Africa. Pan African Med J. 2016;25(18):1-4. doi:10.11604/pamj.2016.25.18.10658

38. Hara L, Guirguis R, Hummel K, Villanueva M. More than bar codes: integrating global standards-based bar code technology into national health information systems in Ethiopia and Pakistan to increase end-to-end supply chain visibility. Glob Heal Sci Pract. 2017;5 (4):678-685. doi:10.9745/GHSP-D-16-00350

39. Harapko S. How COVID-19 impacted supply chains and what comes next. Ernst \& Young; 2021:1-10. Available from: https://www.ey. com/en_gl/supply-chain/how-covid-19-impacted-supply-chains-andwhat-comes-next. Accessed December 21, 2021.

covers a very wide range of areas and welcomes submissions from practitioners at all levels, from all over the world. The manuscript management system is completely online and includes a very quick and fair peer-review system. Visit http://www.dovepress.com/testimonials. php to read real quotes from published authors. 